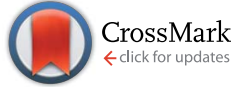

Cite this: RSC Adv., 2016, 6, 71370

Received 19th June 2016

Accepted 30th June 2016

DOI: $10.1039 / c 6 r a 15884 f$

www.rsc.org/advances

\section{Flexible thermo-electrochemical cells using lodolyte HI-30 for conversion of low-grade heat to electrical energy}

\author{
Zubair Ahmad, ${ }^{* a}$ Jolly Bhadra, ${ }^{\text {b }}$ Farid Touati, ${ }^{a}$ Abdullah Alashraf, ${ }^{b}$ R. A. Shakoor ${ }^{b}$ \\ and N. J. Al-Thani ${ }^{\text {b }}$
}

In this research work, we investigated the flexible thermo-electro chemical cells (TECs) for the conversion of low-grade heat into electrical energy in the temperature range of $20-70{ }^{\circ} \mathrm{C}$. The TECs were fabricated using $30 \mathrm{mM}$ iodide/tri-iodide (lodolyte $\mathrm{HI}$-30) in acetonitrile as a redox couple. A TEC consists of a carbon/ lodolyte $\mathrm{HI}-30 /$ carbon structure. The cell contains a flexible polymer tube, filled with the electrolyte, whereas the openings of the tube have been sealed using carbon electrodes. Gradients of temperature $(\Delta T)$ up to $50{ }^{\circ} \mathrm{C}$ have been created. The Seebeck voltages and short-circuit currents $\left(I_{\mathrm{sc}}\right)$ were found to be $8-17 \mathrm{mV}$ and $100-425 \mu \mathrm{A}$, respectively, in the $20-70{ }^{\circ} \mathrm{C}$ temperature range. It was found that the TECs could provide sufficient power to drive low-power electronic devices and could be used for measurement of temperature gradients as well. Furthermore, this work identifies the directions for realizing flexible thermo-electric cells that can be potentially used for several applications in the medical segment where, besides electrical and mechanical stability, flexibility is paramount.

\section{Introduction}

In order to meet the increasing energy requirements of the future, it is very important to seek alternative energy sources. The emerging trend of green renewable energy has motivated researchers to develop thermo-electric materials that could lead to eco-friendly and economical thermo-electric devices. Thermo-electric generators (TEGs) can convert waste heat to electricity, which is the major advantage of this technology. The preliminary research work on TEGs was performed on inorganic semiconductors, ${ }^{\mathbf{1}}$ which provide high values of the figure of merit (ZT). ${ }^{2}$ Moreover, to enhance the $\mathrm{ZT}$ for the inorganic materials, the materials have been tailored by several approaches, such as nanostructured skutterudites and halfHeusler alloys, and a ZT up to 2.4 was found. ${ }^{3}$ By using these approaches, the thermal conductivity of the inorganic materials can be reduced while maintaining the electrical conductivity, which results in the enhanced performance of the TEGs. To develop more environmentally friendly and economical TEGs, researchers' attention has been diverted from inorganic to organic semiconductor materials. The highest ZT that has been achieved was 2-3 orders of magnitude lower than those of inorganic TE materials. However, these materials have several advantages, such as flexibility, light weight, controllable size and low cost. ${ }^{4,5}$

${ }^{a}$ Department of Electrical Engineering, College of Engineering, Qatar University, Doha 2713, Qatar. E-mail: zubairtarar@qu.edu.qa; Tel: +97466461595

${ }^{b}$ Center for Advanced Materials, Qatar University, Qatar, Doha 2713, Qatar
On the other hand, ionic liquid based thermo-electrochemical cells can provide continuous and economical operation at moderate temperature, produce no carbon emissions and have low operation costs. ${ }^{6}$ Besides the non-flammable nature of the ionic liquids, their thermal and electro-chemical stability make them very favorable candidates (as electrolytes) for applications involving low-grade heat. Further, ionic liquid-based devices have lengthier service lifespans owing to their small vapor pressure coefficients. The potential of ionic liquids for low temperature thermal energy applications has already been reported in detail by many researchers. ${ }^{6-10}$ Low-grade heat, below $200{ }^{\circ} \mathrm{C}$, can be efficiently converted into electrical energy by ionic liquid-based thermo-electrochemical cells. Bonetti et al. ${ }^{8}$ reported that solutions of tetraalkylammonium ions in soluble organic solvents (octanol, dodecanol, ethylene-glycol, etc.) reach exceptionally large Seebeck coefficients $(\alpha)$ in the order of $10 \mathrm{mV} \mathrm{K}^{-1}$. This is the highest Seebeck coefficient $\alpha$ that has been observed so far in nonaqueous thermo-electric cells. The low power obtained from these thermo-electric generators has a number of applications, especially in medical wearable devices where the energy requirement lies between $10 \mu \mathrm{W}$ and $5 \mathrm{~mW}$. Some other applications of such thermo-electric generators could be cardiac pacemaker, drug pumps, glucose monitors, etc.

Keeping in view the recently reported potential of ionic liquids in thermo-electrochemical cells, in the presented work the ionic liquid "Iodolyte HI-30" is used as an electrolyte for the fabrication of thermo-electrochemical cells. Iodolyte HI-30 is well known as a high-performance non-aqueous ionic electrolyte. It is typically used for the best performance in Dye 


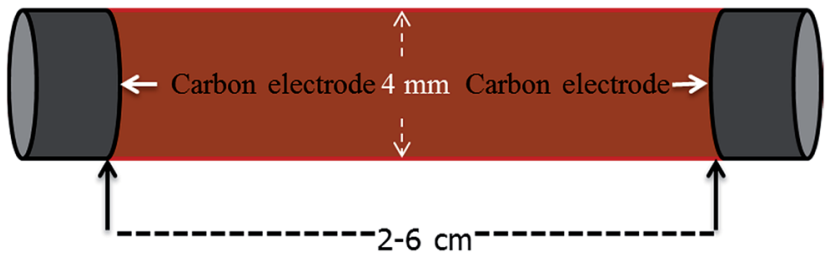

Fig. 1 Schematic diagram of the flexible carbon/lodolyte HI-30/ carbon thermo-electric cell.

Sensitized Solar Cells (DSSCs). In the present study, this has been used to serve the purpose of the electrolyte in a thermogalvanic cell. The aim of our present research is to use Iodolyte $\mathrm{HI}-30$ as an electrolyte to make flexible, low-cost thermogalvanic cells and to study their performance under various circumstances.

\section{Methods}

The carbon/Iodolyte HI-30/carbon thermogalvanic cells were fabricated using commercially available Iodolyte HI-30 and flexible polymer tubes. The flexible polymer tubes were made of polyethylene. Iodolyte $\mathrm{HI}-30$ was used as an electrolyte. Iodolyte HI-30 is a brownish colored liquid that consists of iodide/triiodide as a redox couple (redox concentration $0.03 \mathrm{M}$ ) and additives, i.e., ionic liquid, lithium salt and pyridine derivative. Iodolyte $\mathrm{HI}-30$ is a high-performance electrolyte that helps maximize the performance of dye-sensitized solar cells, which has been proven in real solar cells. The TGC cells were fabricated using flexible polymer cylindrical tubes. The internal diameter, external diameter of the tube and the surface area of the electrodes were fixed as $4 \mathrm{~mm}, 6 \mathrm{~mm}$ and $0.126 \mathrm{~cm}^{2}$, respectively. The samples were prepared with three different lengths, i.e. 2, 4 and $6 \mathrm{~cm}$. Fig. 1 shows the schematic diagram of the flexible carbon/Iodolyte HI-30/carbon thermogalvanic cell. Device voltages and currents were measured by SMU. Temperature gradients have been created by oil bath and measured by thermocouples. During the measurements, the temperature of the cold side of the cell was kept constant $(20 \pm$ $1{ }^{\circ} \mathrm{C}$ ), whereas the temperature of the hot side was dependent on the oil temperature. To guarantee reproducibility, experimental measurements were recorded three times and the subsequent error was found to be $\pm 1.85 \%$. The temperaturedependent electrical and thermal conductivities were measured by Novocontrol broadband dielectric spectrometer and thermal conductivity measurement unit (Hot Disk TPS 2500), respectively.

\section{Results and discussion}

Fig. 2 shows the relations between the Seebeck coefficient $(\alpha)$, short-circuit current $\left(I_{\mathrm{sc}}\right)$, and gradient of temperature $(\Delta T)$ for the carbon/Iodolyte $\mathrm{HI}-30 /$ carbon thermo-electric cell. It is observed that $(\alpha)$ and $I_{\mathrm{sc}}$ increase with the rise in temperature gradient $(\Delta T)$. This could be explained by the theory of thermoelectric cells, where the thermo-electric voltage is directly

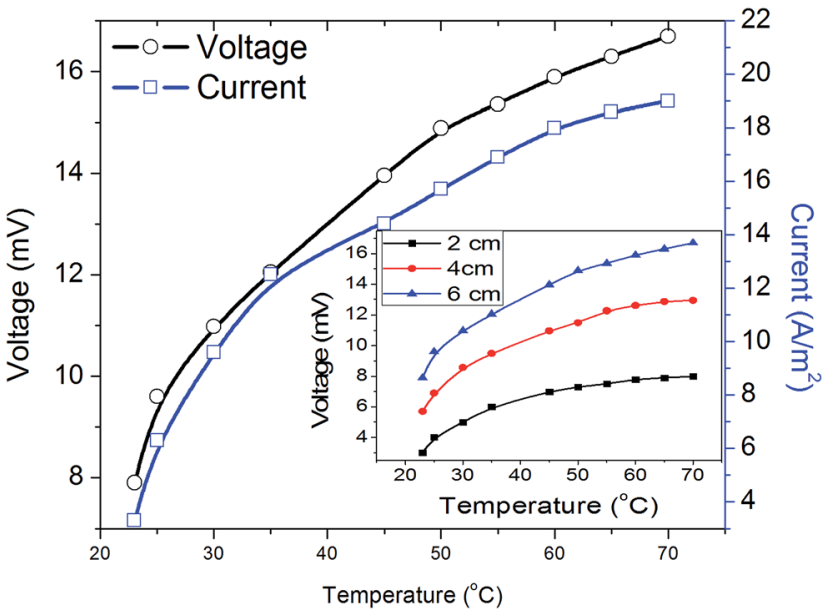

Fig. 2 Seebeck coefficient $(\alpha)$, and temperature dependence of shortcircuit currents $\left(I_{\mathrm{sc}}\right)$ of the carbon/lodolyte $\mathrm{HI}-30$ (redox concentration $0.03 \mathrm{M}) /$ carbon thermogalvanic cells. Inset shows the open circuit voltages for different lengths of the TEG devices as a function of hot electrode temperature.

proportional to the temperature gradient between both junctions of the thermo-electric generators ${ }^{11,12}$ and can be expressed by the relation $V_{\mathrm{oc}}=m \alpha \Delta T$ where $m$ is the number of thermocouples in the thermo-electric unit, $\alpha$ is the relative Seebeck coefficient of the materials and $\Delta T$ is the temperature difference between the cold and hot junctions of the thermo-electric device. In the presence of a temperature difference between the hot and cold sides, the thermo-electric voltages are developed as a result of drift of charges. In the case of the solutionbased thermo-electric (thermogalvanic) cells, the thermoelectric behavior is more complex as compared to the solidstate thermo-electric generators. The electric current in the thermogalvanic cells is owing to ionic flow. The ionic flow takes place owing to roaming of molecules and ions that could easily drift through when a potential difference is produced by creating a temperature gradient between the electrodes.

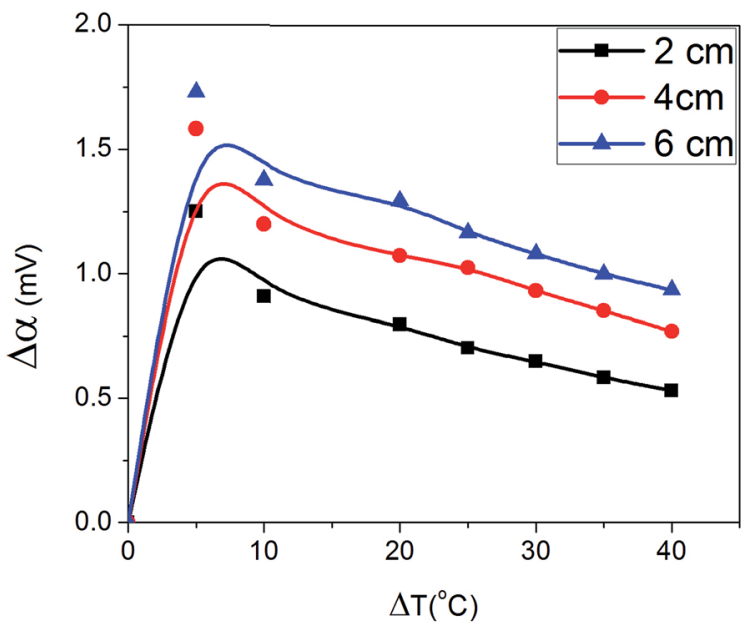

Fig. 3 Temperature dependence of change in the Seebeck coefficient of the carbon/lodolyte $\mathrm{HI}-30 / c a r b o n$ thermogalvanic cell. 


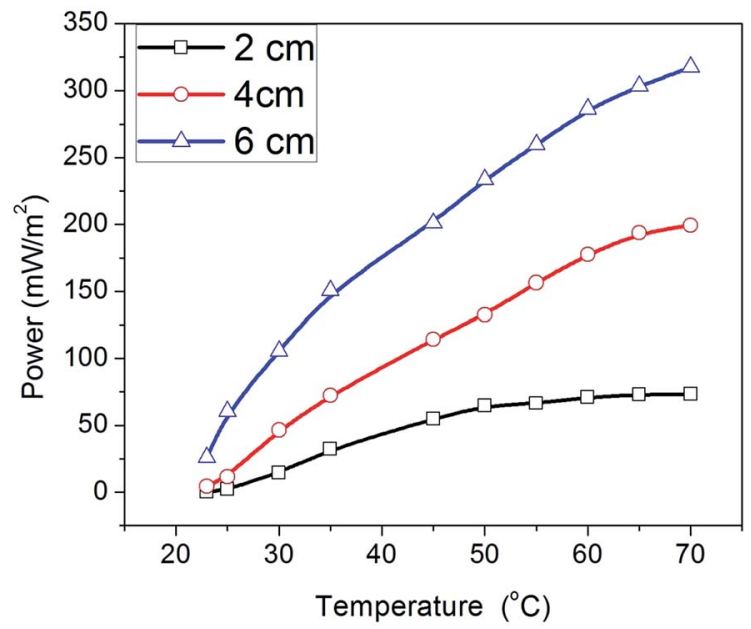

Fig. 4 Temperature-dependent electric power output of the carbon/ lodolyte $\mathrm{HI}-30 /$ carbon thermo-electric cell.

Therefore, it can be supposed that the variation of the entropy might bring about the particular changes of the thermo-electric coefficient. From a thermodynamic point of view, it can be supposed that the reaction entropy $\left(\Delta S_{\mathrm{rc}}\right)$ of the redox couple controls the temperature reliance of the electrode potential and can be expressed by the equation $n F \Delta S_{\mathrm{rc}}=\partial E(T) / \partial T$, where $E(T)$ represents the electrode potential of the redox couple, $n$ is the number of electrons involved in the redox reaction and $F$ is Faraday's constant. ${ }^{13}$ The Seebeck coefficient is associated with the structural changes of the redox types in addition to the adjacent solvent during the redox procedure. It can be undertaken that in the case of HI-30 thermal energy will bring a significant upsurge in the entropy owing to the presence of the redox couple, which can cause the ion mobility from the hot to the cold side. In the inset of Fig. 2, it is also found that the Seebeck coefficient depends upon the length of the tube. Therefore, for different $\Delta T$, the different sizes (dimensions) of the TECs can be designed in order to achieve the maximum power for different applications.

Fig. 3 shows the temperature dependence of the change in Seebeck coefficient $(\Delta \alpha)$ of the carbon/Iodolyte HI-30/carbon thermogalvanic cell. Experimentally attained $\Delta \alpha$ values are in the range of $0.2-0.8 \mathrm{mV}^{\circ} \mathrm{C}^{-1}$, which is comparable to the $\alpha$ of the traditional and even new thermo-electric materials $\mathrm{C}_{60}$, plasma-treated CNTs and graphene $\left(190-390 \mu \mathrm{V}{ }^{\circ} \mathrm{C}^{-1}, 350 \mu \mathrm{V}\right.$ ${ }^{\circ} \mathrm{C}^{-1}$ and $700 \mu \mathrm{V}{ }^{\circ} \mathrm{C}^{-1}$, respectively). ${ }^{14}$ Theoretically, $\alpha$ should not be temperature-dependent, therefore the relation between $\alpha$ and temperature should be linear. However, practically, it cannot be linear because the electrolyte is at a transient state while heating. The correct way of determining the $\alpha$ is to fix the $\Delta T$ and measure the steady-state voltage to avoid any transient state heating effect. The electrochemical reactions of metal hydrides electrodes with electrolyte combinations such as ( $\mathrm{LiCl}-\mathrm{KCl}-\mathrm{LiH}$ ) have been investigated by Murakami et al. ${ }^{15} \mathrm{It}$ was found that metal hydride electrodes have the ability to yield large voltages, but such systems face the problem of embrittlement of the electrodes during cell operation. It has been reported in ref. 16 that solutions, such as iodide/triiodide, in association with platinum electrodes could be an advantageous choice because of their small vapor pressure, high thermal stability, and nonflammable nature, which should foster longlifetime thermogalvanic devices. This experiment was performed in a number of ionic liquids (up to $150{ }^{\circ} \mathrm{C}$ ). In order to fully understand the potential of ionic liquids in thermogalvanic devices, further research work is very much desirable in a number of dimensions, such as: study of a broader range of redox couples to find those with large values of reaction entropy, more investigation on the entropy change in ionic liquids and searching for alternative electrode/electro-catalytic materials to optimize surface kinetics and reduce cost.

The power curve shown in Fig. 4 demonstrates the maximum output power capacity of the thermal-electric generator over the temperature gradient $(\Delta T)$ range from 0 to $50{ }^{\circ} \mathrm{C}$. The output power ranges from hundreds of $\mu \mathrm{W}$ to tens of $\mathrm{mW}$ over this temperature range. Almost $300 \mathrm{~mW} \mathrm{~m}^{-2}$ of energy is produced from a temperature difference of $50{ }^{\circ} \mathrm{C}$. In order to prove this cell for low-power medical applications, the out power of the cell was investigated at $\Delta T \sim 15{ }^{\circ} \mathrm{C}$. This temperature gradient was attained when one electrode was in contact with a human body and other was exposed to room temperature. A single unit cell with the dimensions given in this work can produce $15 \mu \mathrm{W}$ $\mathrm{cm}^{-2}$ at room temperature for $\Delta T \sim 15{ }^{\circ} \mathrm{C}$. However, the maximum power at any certain Seebeck coefficient depends upon the length of the cell and the temperature gradient. The $V_{\text {oc }}$ of the carbon/Iodolyte HI-30/carbon thermogalvanic cell was compared with the other ionic liquid based thermo-electric cells reported in the literature, as shown in Table 1. A typical lowpower application, such as medical wearable devices, requires in the region of hundreds of $\mathrm{nW}$ to operate the devices. Nonetheless, in practice, before applying it to a medical device, the thermo-electric voltage has to be boosted to higher levels using a DC-DC boost converter. This process results in conversion losses that would require a higher generated power than what is required to compensate for the conversion loss. The proposed

Table 1 Comparison of the potential difference of the carbon/lodolyte $\mathrm{HI}-30 /$ carbon thermo-electric cell with other ionic liquids at $\Delta T=15^{\circ} \mathrm{C}$

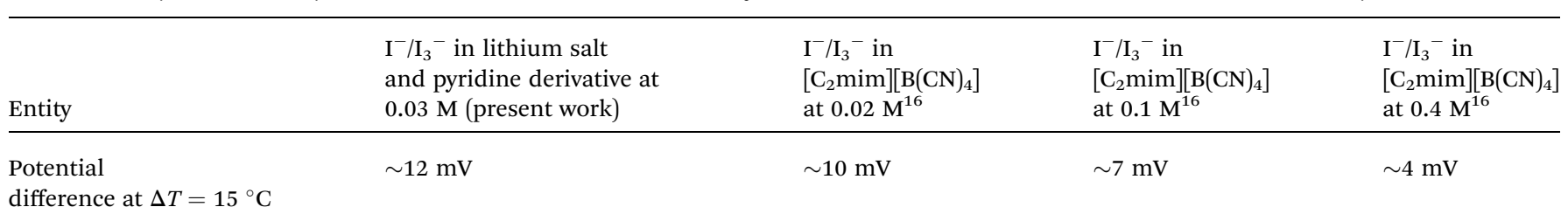

difference at $\Delta T=15{ }^{\circ} \mathrm{C}$ 
devices need hundreds of $\mathrm{nW}$; the rest is enough to cover the booster losses or alternatively it can be stored in a storage device (e.g. capacitor) for future use. It is important to note that this maximum output power capacity assumes an ideal load match without any conversion loss. The power curve of the TECs is highly temperature-dependent, as given in the expression $P_{\mathrm{o}}=$ $\left(m^{2} \alpha^{2} / 4 R_{\mathrm{D}}\right) \Delta{T_{\mathrm{G}}}^{2}:^{12}$ where $R_{\mathrm{D}}$ is the internal electrical resistance of the TEC. By using the impedance analyzer study, the internal electrical resistance $\left(R_{\mathrm{D}}\right)$ of the TE device has been predicted in $\mathrm{k} \Omega$. The resistance of carbon electrodes is less than an ohm $(1 \Omega)$. Overall, the proposed thermo-electric cell generates an output electrical power in the range between $25 \mathrm{~mW} \mathrm{~m}^{-2}$ to $300 \mathrm{~mW} \mathrm{~m}^{-2}$ in the temperature difference range 5 to $50{ }^{\circ} \mathrm{C}$.

The power conversion efficiency of thermo-electric cells depends not only on the Seebeck coefficient $(\alpha)$ but also on the electrical/ionic and thermal conductivities of the cell. ${ }^{17}$ Although solution-based thermo-electric cells have shown very promising values of $\alpha$, the electrical conductivity of the cell hinders the maximum power output of such cells. The electrical conductivity of the iodide/tri-iodide (redox concentration $30 \mathrm{mM}$ in acetonitrile) as a function of temperature is shown in Fig. 5. It is seen that the electrical conductivity tends to decrease with the increment of temperature. This may be owing to the fact that, as the temperature rises, the thermal-electric motion interrupts the smooth ionic flow. Besides the electrical conductivity, the thermal conductivity is a key parameter that determines the thermal to electrical energy conversion efficiency of the thermo-electric generators. The stated values of the thermal conductivity of the ionic liquids are typically lower than those of aqueous solutions. ${ }^{9}$ For instance, the thermal conductivity for Iodolyte HI-30 $\left(\sim 0.2 \mathrm{~W} \mathrm{~m}^{-1} \mathrm{~K}^{-1}\right)$ is about three times lower than that of water $\left(\sim 0.6 \mathrm{~W} \mathrm{~m}^{-1} \mathrm{~K}^{-1}\right)$. This makes it more beneficial for thermo-electric applications because the low thermal conductivity of a material overwhelms the thermal energy loss across the thermo-electric device and permits

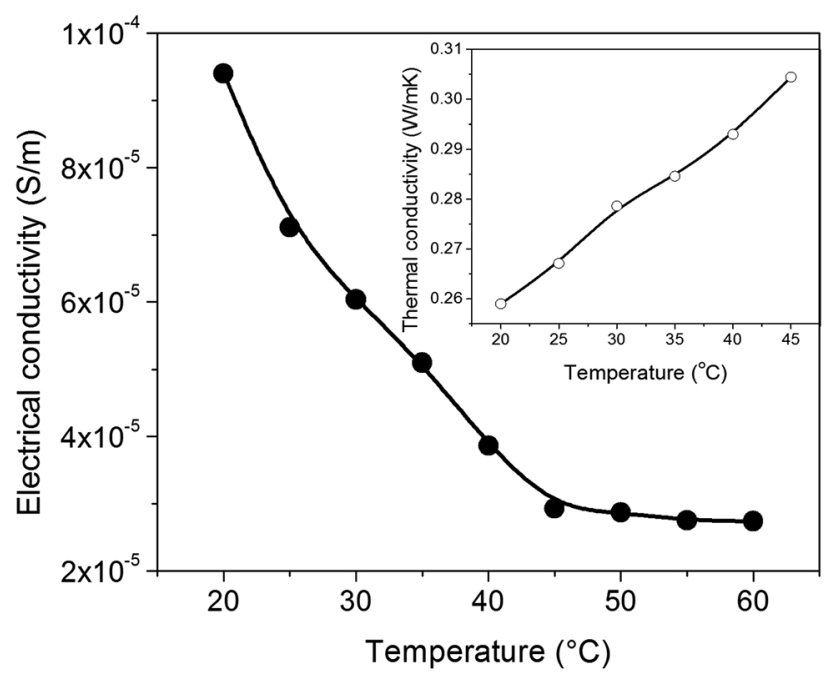

Fig. 5 Electrical conductivity of the lodolyte $\mathrm{HI}-30$ solution as a function of temperature. Inset shows the thermal conductivity vs. temperature curve.

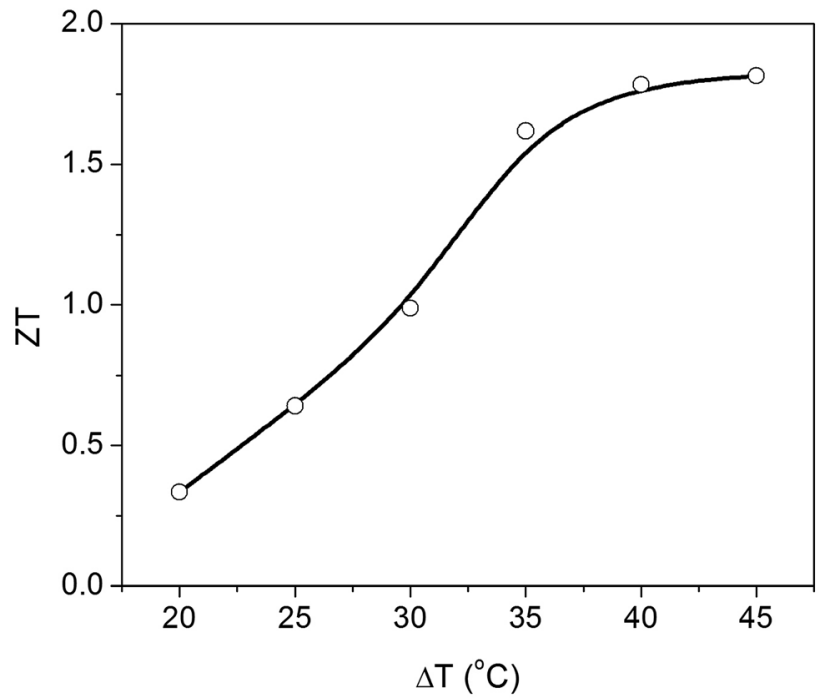

Fig. 6 Figure of merit for the carbon/lodolyte HI-30/carbon thermoelectric cell.

a greater temperature gradient to be retained between the heat source and heat sink electrodes.

The efficiency of a thermo-electric device strictly depends upon the properties of the semiconductor materials. Ideally, a good thermo-electric device possesses minimum thermal conductivity to sustain a high temperature gradient, along with having maximum electrical conductivity and Seebeck coefficient. The efficiency of a thermo-electric is usually described by its figure of merit (ZT), which is a dimensionless unit that depends on the Seebeck coefficient $(\alpha)$, absolute temperature $(T)$, electrical conductivity $(\sigma)$, and thermal conductivity $(\kappa)$ : ZT $=\sigma \alpha^{2} T / \kappa$. ZT values calculated for the TECs fabricated in this work are shown in Fig. 6. It is observed that the ZT value tends to incline with increasing temperature. As said above, ZT requires high electrical conductivity but low thermal conductivity. ${ }^{18}$ However, it is important to note that in the case of solution-based thermo-electric systems, electrical conductivity $(\sigma)$ is neither essentially constant nor ohmic in nature. ${ }^{7}$ Thus, it is important to calculate only $Z$, when defining the power efficiency of solution based thermo-electric devices.

\section{Conclusion}

Iodolyte HI-30 has been investigated as an electrolyte for the fabrication of flexible thermo-electric cells (TECs). The TECs showed a good response towards the conversion of low grade heat $\left(20-70{ }^{\circ} \mathrm{C}\right)$ into electric power. The TECs could provide sufficient power to drive low-power electronic devices and could be used for the measurement of temperature gradients as well. The TECs epitomize an important approach forward in the development of flexible thermo-electric energy-producing devices.

\section{Conflict of interest}

The authors declare no competing financial interests. 


\section{Acknowledgements}

This publication was made possible by PDRA grant no. PDRA10117-14109 from the Qatar National Research Fund (a member of the Qatar Foundation). The findings achieved herein are solely the responsibility of the authors.

\section{References}

1 F. J. Congcong Liu, M. Huang, B. Lu, R. Yue and J. Xu, J. Electron. Mater., 2011, 40, 948.

2 H. D. Manfred Scholdt, J. Lang, A. Gall, A. Colsmann, U. Lemmer, J. D. Koenig, M. Winkler and H. Boettner, J. Electron. Mater., 2010, 39, 1589-1592.

3 R. Venkatasubramanian, E. Siivola, T. Colpitts and B. O'Quinn, Nature, 2001, 413, 597-602.

4 J. S. B. Zhang, H. E. Katz, F. Fang and R. l. Opila, ACS Appl. Mater. Interfaces, 2010, 2, 3170-3178.

5 Q. Jiang, C. Liu, J. Xu, B. Lu, H. Song, H. Shi, Y. Yao and L. Zhang, J. Polym. Sci., Part B: Polym. Phys., 2014, 52, 737742.

6 T. J. Abraham, D. R. MacFarlane, R. H. Baughman, L. Jin, N. Li and J. M. Pringle, Electrochim. Acta, 2013, 113, 87-93.

7 A. Gunawan, C.-H. Lin, D. A. Buttry, V. Mujica, R. A. Taylor, R. S. Prasher and P. E. Phelan, Nanoscale Microscale Thermophys. Eng., 2013, 17, 304-323.
8 M. Bonetti, S. Nakamae, M. Roger and P. Guenoun, J. Chem. Phys., 2011, 134, 114513.

9 D. R. MacFarlane, N. Tachikawa, M. Forsyth, J. M. Pringle, P. C. Howlett, G. D. Elliott, J. H. Davis, M. Watanabe, P. Simon and C. A. Angell, Energy Environ. Sci., 2014, 7, 232-250.

10 T. Migita, N. Tachikawa, Y. Katayama and T. Miura, Electrochemistry, 2009, 77, 639-641.

11 J. Y. Xing Niu and S. Wang, J. Power Sources, 2009, 188, 621626.

12 W. Glatz and C. H. Simon Muntwyler, Sens. Actuators, A, 2006, 132, 337-345.

13 J. T. Hupp and M. J. Weaver, Inorg. Chem., 1984, 23, 36393644.

14 Z. Ahmad, K. S. Karimov, N. Fatima and F. Touati, J. Mater. Sci.: Mater. Electron., 2016, 27, 2442-2447.

15 T. Murakami, T. Nishikiori, T. Nohira and Y. Ito, J. Electrochem. Soc., 2003, 150, A928-A932.

16 T. J. Abraham, D. R. MacFarlane and J. M. Pringle, Chem. Commun., 2011, 47, 6260-6262.

17 C.-H. Lin, A. Gunawan, P. E. Phelan, D. A. Buttry, V. Mujica, R. A. Taylor and R. Prasher, ASME International Mechanical Engineering Congress and Exposition, 2012, pp. 541-547.

18 G. J. Snyder and E. S. Toberer, Nat. Mater., 2008, 7, 105-114. 